\title{
AN EQUIVALENCE FOR THE EMBEDDINGS OF CELLS IN A 3-MANIFOLD
}

\author{
BY \\ R. J. DAVERMAN( $\left.{ }^{1}\right)$ AND W. T. EATON( $\left.{ }^{1}\right)$
}

1. Introduction. In studying the embedding of a topological cell $C$ in a manifold $M$, it is useful to know whether a lower dimensional cell $C^{\prime}$ has an embedding (in $M$ ) which is similar, under some reasonable definition of the word, to that of $C$. In this paper we show that if $C$ is an $i$-cell in the interior of a 3-manifold $M$ and $j$ is a positive integer less than $i$, then there exist a $j$-cell $D$ in $M$ and a map $f$ of $M$ onto itself such that $f(C)=D$ and $f$ is a homeomorphism of $M-C$ onto $M-D$; furthermore, the map $f$ restricted to $C$ acts formally like a projection map, and $f$ is the identity map outside a preassigned neighborhood of $C$.

In case that $C$ is a cell in the interior of a 3-manifold $M$ and $D$ is a cell in $\mathrm{Bd} C$ such that $C$ is locally tame at points of $C-D$, then well-known techniques provide such a map collapsing $C$ onto $D$. Otherwise, the facts established about this problem concern the related question for decomposition spaces; namely, if $C$ is a cell in Int $M$, is there a lower dimensional cell $C^{\prime}$ in Int $M$ such that $M / C$ and $M / C^{\prime}$ are homeomorphic? For example, Armentrout [3] and Meyer [17] have shown that for special embeddings of a 3-cell $C$ in $E^{3}$ there is an arc $A$ in $E^{3}$ such that $E^{3} / A$ is homeomorphic to $E^{3} / C$. Armentrout, Lininger, and Meyer [4] have also proved that if $C$ is a tamely finnable 3 -cell in $E^{3}$, there is a 2-cell $D$ in $E^{3}$ such that $E^{3} / D$ and $E^{3} / C$ are homeomorphic. Corollary 5 is an extension of these results to arbitrary embeddings of a 3-cell $C$ in $E^{3}$.

The main results of this paper are stated in $\$ 3$, although the proof of one of the theorems, involving some intricate geometry and epsilonics, is delayed until $\S 4$. In $\S 5$ we discuss extensions of the results of $\S 3$ to embeddings of a cube with handles in the interior of a 3-manifold.

2. Definitions and notation. We use $\Delta_{1}, \Delta_{2}$, and $\Delta_{3}$ to denote standard 1,2, and 3-cells defined by $\Delta_{3}=\left\{(x, y, z) \mid x^{2}+y^{2}+z^{2} \leqq 1\right\}, \Delta_{2}=\left\{(x, y, 0) \mid x^{2}+y^{2} \leqq 1\right\}$ and $\Delta_{1}=\{(x, 0,0) \mid-1 \leqq x \leqq 1\}$. We use $\rho$ to denote the projection map of $\Delta_{3}$ onto $\Delta_{2}$ taking $(x, y, z)$ to $(x, y, 0)$ and $\pi$ to denote the projection map of $\Delta_{2}$ onto $\Delta_{1}$ taking $(x, y, 0)$ to $(x, 0,0)$.

Let $M$ be a 3-manifold (possibly with boundary). We use $d$ to denote a metric on $M$. If $A$ is a subset of $M$ and $\delta$ is a positive number, then $N(A, \delta)$ denotes the set

Received by the editors September 27, 1968.

( ${ }^{1}$ ) This paper supported in part by NSF Grants GP-5420 and GP-8888.

Copyright (C) 1969, American Mathematical Society 
of points of $M$ whose distance from $A$ is less than $\delta$, and Diam $A$ denotes the diameter of $A$.

An $n$-frame $G_{n}$ is the union of $n$ arcs $A_{1}, \ldots, A_{n}$ with a distinguished point $p$ such that $p$ is an endpoint of each $A_{i}$ and $A_{i} \cap A_{j}=p$ for $i \neq j$. The boundary of $G_{n}$, denoted $\mathrm{Bd} G_{n}$, is $\bigcup\left(\mathrm{Bd} A_{i}-p\right)$.

If $X$ is a metric space and $C$ is a closed subset of $X$, then $X / C$ designates the decomposition space associated with the upper semicontinuous decomposition of $X$ whose only nondegenerate element is $C$.

The unit interval $[0,1]$ is denoted by 1 .

3. Equivalent embeddings. McMillan [16] has shown that any cell embedded in the interior of a 3-manifold has a neighborhood that can be embedded in $E^{3}$. Consequently, in the proofs of all the theorems in this section we may assume that the 3-manifold $M$ is $E^{3}$.

Theorem 1. Suppose that $K$ is a 3-cell in the interior of a 3-manifold $M$ and that $U$ is an open subset of $M$ with $K \subset U$. Then there exist a map fof $M$ onto $M$, a homeomorphism $g$ of $\Delta_{3}$ onto $K$, and a homeomorphism $h$ of $\Delta_{2}$ onto $f(K)$ such that

(1) $f$ is a homeomorphism of $M-K$ onto $M-f(K)$,

(2) $f \mid M-U=$ identity,

(3) $f g=h \rho$.

Proof. Let $J$ be a simple closed curve in $\mathrm{Bd} K$, and let $D_{1}$ and $D_{2}$ be the disks in Bd $K$ bounded by $J$. By the techniques of [11] there is a map $f$ of $M-\operatorname{Int} K$ onto $M$ such that

(1) $f$ is a homeomorphism of $M-K$ onto $M-f\left(D_{1}\right)$,

(2) $f \mid M-U=$ identity,

(3) $f\left(D_{1}\right)=f\left(D_{2}\right)$,

(4) $f \mid D_{i}$ is a homeomorphism $(i=1,2)$.

The map $h$ may be any homeomorphism of $\Delta_{2}$ onto $f\left(D_{1}\right)$, and it is then a simple matter to extend $f$ over all of $M$ and to obtain the required homeomorphism $g$.

The proof of the following theorem is discussed in $\$ 4$.

THEOREM 2. Suppose that $F$ is a 2-cell in the interior of a 3-manifold $M$ and that $U$ is an open subset of $M$ with $F \subset U$. Then there exist a map fof $M$ onto $M$, a homeomorphism $g$ of $\Delta_{2}$ onto $F$, and a homeomorphism $h$ of $\Delta_{1}$ onto $f(F)$ such that

(1) $f$ is a homeomorphism of $M-F$ onto $M-f(F)$,

(2) $f \mid M-U=$ identity,

(3) $f g=h \pi$.

THEOREM 3. Suppose that $K$ is a 3-cell in the interior of a 3-manifold $M$ and that $U$ is an open subset of $M$ with $K \subset U$. Then there exist a map $f$ of $M$ onto $M$, a homeomorphism $g$ of $\Delta_{3}$ onto $K$, and a homeomorphism $h$ of $\Delta_{1}$ onto $f(K)$ such that

(1) $f$ is a homeomorphism of $M-K$ onto $M-f(K)$,

(2) $f \mid M-U=$ identity,

(3) $f g=h \pi \rho$. 
Proof. Apply Theorem 1 to obtain a map $f_{1}$ of $M$ onto itself that takes $K$ onto a 2-cell. Then, by Theorem 2, there exist a map $f_{2}$ of $M$ onto itself, a homeomorphism $g_{2}$ of $\Delta_{2}$ onto $f_{1}(K)$, and a homeomorphism $h$ of $\Delta_{1}$ onto $f_{2} f_{1}(K)$ such that $f_{2}$ is a homeomorphism of $M-f_{1}(K)$ onto $M-f_{2} f_{1}(K), f_{2} \mid M-f_{1}(U)=$ identity, and $f_{2} g_{2}=h \pi$. Define $f \mid M-$ Int $K=f_{2} f_{1} \mid M-$ Int $K$, and define $g^{\prime}$ to be a homeomorphism of Bd $\Delta_{3}$ onto Bd $K$ such that $f_{1} g^{\prime}=g_{2} \rho$. Hence, $f g^{\prime}=f_{2} f_{1} g^{\prime}=f_{2} g_{2} \rho=h \pi \rho$. To complete the proof, let $g$ be a homeomorphism of $\Delta_{3}$ onto $K$ extending $g^{\prime}$, and let $f \mid$ Int $K=h \pi \rho g^{-1} \mid$ Int $K$.

COROLlaRY 4. If $K$ is a cell in the interior of a 3-manifold $M$, then there exists an $\operatorname{arc} A$ in $M$ such that $M-A$ is homeomorphic to $M-K$.

COROLlaRY 5. If $K$ is a cell in the interior of a 3-manifold $M$, then there exists an arc $A$ in $M$ such that $M / A$ is topologically $M / K$. Furthermore, if $K$ is not a 1-cell then there exists a disk $D$ in $M$ such that $M / D$ is topologically $M / K$.

From Corollary 5 and the theorem by Andrews and Curtis [2] we obtain another proof of a result due to Bryant [9].

CoROllaRY 6. If $K$ is a cell in $E^{3}$, then $E^{3} / K \times E^{1}$ is homeomorphic to $E^{4}$.

The next two corollaries follow from Corollary 5 and Kwun's extension [14] of the Andrews and Curtis theorem.

CoROllary 7. If $K_{1}$ and $K_{2}$ are cells in $E^{3}$, then $E^{3} / K_{1} \times E^{3} / K_{2}$ is homeomorphic to $E^{6}$.

Corollary 8. If $K$ is a cell in $E^{3}$ and $\alpha$ is an arc in $E^{n}$, then $E^{n} / \alpha \times E^{3} / K$ is homeomorphic to $E^{n+3}$.

REMARKS. The tameness of a cell is preserved by this squeezing process, for if $K$ is a 3-cell in Int $M$ and $f$ is a map of $M$ onto itself that satisfies the conclusions of Theorem 1, then well-known results such as Bing's 1-ULC Criterion [6] imply that $f(K)$ is tame; if $K$ is a tame cell in Int $M$ and $f$ is a map of $M$ onto itself that satisfies the conclusions of either Theorem 2 or Theorem 3, then Theorem 1 of [8] implies that $f(K)$ is tame.

However, if $K$ is a wild cell, the images of $K$ under two different projection maps may be inequivalently embedded. For example, if $K$ is the 3-cell described in $\$ 2$ of [1], there is a map $f_{1}$ of $E^{3}$ onto itself satisfying the conclusions of Theorem 3 that collapses $K$ onto the arc $W$ of points in $\mathrm{Bd} K$ where $\mathrm{Bd} K$ is wild, and, therefore, $f_{1}(K)$ is a wild arc; there is another $\operatorname{map} f_{2}$ of $E^{3}$ onto itself satisfying the conclusions of Theorem 3 such that $f_{2}(W)$ is a point, and it can be shown, using properties of this embedding of $K$, that $f_{2}(K)$ is a tame arc.

The following result, a converse to Theorem 1, indicates that near each disk $F$ in $E^{3}$ there is a 3 -cell $K$ that projects (in the sense of Theorem 1) onto the disk. Of course, it may be impossible for $K$ to contain the disk; therefore, the set of points 
moved by the projection map cannot be restricted to neighborhoods arbitrarily close to $K\left({ }^{2}\right)$.

THEOREM 9. If $h$ is a homeomorphism of $\Delta_{2}$ onto a 2-cell $F$ in the interior of a 3manifold $M$ and $U$ is an open subset of $M$ containing Int $F$, then there exist a homeomorphism $g$ of $\Delta_{3}$ onto a 3-cell $K$ in $\mathrm{Bd} F \cup U$ and a map $f$ of $M$ onto $M$ such that

(1) $f$ is a homeomorphism of $M-K$ onto $M-F$,

(2) $f \mid M-U=$ identity,

(3) $f g=h \rho$.

Proof. There is a deformation retraction $r_{t}(0 \leqq t \leqq 1)$ of $E^{3}$ onto $F$. Let $V$ be a connected open subset of $U$ containing Int $F$ such that if $x \in V$ then $r_{t}(x) \notin \operatorname{Bd} F$ $(0 \leqq t \leqq 1)$. It can be shown that $V$ - Int $F$ is the disjoint union of two open sets $V_{1}$ and $V_{2}$, each of which contains $F$ in its closure.

It follows from techniques of [13] or [15], that for $i=1,2$, there exists a homeomorphism $f_{i}$ of $\mathrm{Cl} V_{i}$ into $\mathrm{Cl} V$ such that $f_{i} \mid \operatorname{Bd} V_{i}-\operatorname{Int} F=$ identity, $f_{i}($ Int $F)$ is locally tame from $V-f_{i}\left(\mathrm{Cl} V_{i}\right)$, and $f_{1}\left(\mathrm{Cl} V_{1}\right) \cap f_{2}\left(\mathrm{Cl} V_{2}\right)=\mathrm{Bd} F$.

Let $S$ be the 2-sphere $f_{1}(F) \cup f_{2}(F)$. Hence, by the construction, $S$ is locally tame from Int $S$ at points of $S-\mathrm{Bd} F$. In order to show that $S$ is locally tame from Int $S$ at points of $\mathrm{Bd} F$, it is sufficient to show that if $x \in \mathrm{Bd} F$ and $\varepsilon>0$, then there exists a positive number $\delta$ such that each simple closed curve in $N(x, \delta) \cap$ Int $S$ can be shrunk to a point in an $\varepsilon$-subset of $E^{3}-\mathrm{Bd} F$.

Let $\alpha$ be a positive number small enough that closed $\alpha$-subsets of Int $F$ lie in $\varepsilon / 3$-disks in Int $F$, and let $x \in \operatorname{Bd} F$. Since $r_{t}(x)=x$ for $0 \leqq t \leqq 1$, there is a positive number $\delta$ such that Diam $\bigcup_{t} r_{t}(N(x, \delta))<\alpha$. If $J$ is a simple closed curve in $N(x, \delta) \cap$ Int $S$, then $J \subset V$ and $r_{t}(J) \cap \mathrm{Bd} F=\varnothing(0 \leqq t \leqq 1)$. It follows that $J$ can be shrunk to a point in an $\varepsilon$-subset of Int $F \cup\left(\bigcup_{t} r_{t}(J)\right)$.

Therefore, $S$ is locally tame from Int $S$, and we let $K$ be $\mathrm{Cl}$ (Int $S$ ). Let $g_{1}$ be a homeomorphism of Bd $\Delta_{3}$ onto $S$ such that $h \rho g_{1}^{-1}(x)=f_{i}^{-1}(x)$ for $x \in f_{i}(F)(i=1,2)$, and let $g$ be a homeomorphism of $\Delta_{3}$ onto $K$ extending $g_{1}$. The desired map $f$ is given by the rule

$$
\begin{aligned}
f(x) & =x & & \text { if } x \in E^{3}-\left(K \cup f_{1}\left(V_{1}\right) \cup f_{2}\left(V_{2}\right)\right) \\
& =f_{1}^{-1}(x) & & \text { if } x \in f_{1}\left(V_{1}\right) \\
& =f_{2}^{-1}(x) & & \text { if } x \in f_{2}\left(V_{2}\right) \\
& =h \rho g^{-1}(x) & & \text { if } x \in K .
\end{aligned}
$$

4. Proof of Theorem 2. We need the following definition.

Definition. A disk $D$ is normally situated in a surface $S$ if $D$ either lies in Int $S$ or intersects $\mathrm{Bd} S$ in an arc. A Sierpiński curve is normally situated in a surface if the closures of the components of its complement are normally situated disks.

${ }^{(2)}$ Theorem 9 was communicated to the first named author by Robert F. Craggs, whose more detailed proof will appear shortly. 
The following lemma is due to Craggs [10, Lemma 5.1].

Lemma 0. Suppose that $M$ is a 3-manifold, $D$ is a disk in $M$, and $\varepsilon$ is a positive number. Then there is a tame Sierpinski curve $X$ in $D$ which is normally situated in $D$ such that each component of $D-X$ has diameter less than $\varepsilon$.

Furthermore if $\left\{X_{j}\right\}$ is a finite collection of sets, each of which is either a tame arc in $D$ or a tame Sierpinski curve normally situated in $D$, then $X$ may be chosen so that $\bigcup_{j} X_{j}$ is contained in the inaccessible part of $X$.

LEMMA 1. Suppose $0 \leqq r<t<s \leqq 1,0<r_{1}<r_{2}<\cdots<r_{k}<1$, $g$ is a homeomorphism of $[r, s] \times I$ into $E^{3}$ such that $g\left([r, s] \times r_{1}\right), \ldots, g\left([r, s] \times r_{k}\right)$, and $g(t \times I)$ are tame, and $U$ is an open set in $E^{3}$ containing $g(t \times I)$. Then there exist positive numbers $r<u_{k}<u_{k-1}<\cdots<u_{1}<t<v_{1}<\cdots<v_{k}<s$, a homeomorphism $\lambda$ of $[r, s] \times I$ onto itself, and a map $\beta$ of $E^{3}$ onto $E^{3}$ such that

(1) $\lambda \mid\{r, t, s\} \times I=$ identity,

(2) $\beta \mid E^{3}-U=$ identity,

(3) $\beta g(t \times I)$ is a point in $U$,

(4) $\beta \mid E^{3}-g(t \times I)$ is a homeomorphism onto $E^{3}-\beta g(t \times I)$.

Furthermore, if $A_{i}=\left(u_{i} \times\left[r_{i}, 1\right]\right) \cup\left(\left[r, u_{i}\right] \times r_{i}\right), B_{i}=\left(v_{i} \times\left[r_{i}, 1\right]\right) \cup\left(\left[v_{i}, s\right] \times r_{i}\right), \delta$ is the diameter of the largest component of $g\left(([r, s] \times I)-\left[\left(I \times\left\{r_{1}, \ldots, r_{k}\right\}\right) \cup(t \times I)\right]\right)$, $\delta^{\prime}$ is the diameter of the largest component of $([r, s] \times I)-\left[\left(I \times\left\{r_{1}, \ldots, r_{k}\right\}\right) \cup(t \times I)\right]$, and $C$ is a component of $([r, s] \times I)-\bigcup_{i}\left(A_{i} \cup B_{i} \cup(t \times I)\right)$ then

(5) $d(x, \lambda(x))<\delta^{\prime}$,

(6) $\operatorname{Diam} \beta g \lambda(C)<5 \delta$,

(7) the $\operatorname{arcs} g \lambda\left(A_{i}\right)$ and $g \lambda\left(B_{i}\right)$ are tame.

Proof. Using Lemma 0 it is straightforward to show that there exist a Sierpiński curve $X \subset[r, s] \times I$ and a homeomorphism $h$ of $E^{3}$ onto $E^{3}$ which moves no point outside a compact subset of $E^{3}$ such that

(8) $X$ is normally situated in $[r, s] \times I$,

(9) $[r, s] \times r_{i}$ and $t \times I$ lie in the inaccessible part of $X$,

(10) $h g(X)$ lies in the $x y$-plane and the outer boundary component of $h g(X)$ is the rectangle with vertices $( \pm 1,0,0)$ and $( \pm 1, k+1,0)$,

(11) $h g(t \times I)=\{(x, y, z) \mid x=0,0 \leqq y \leqq k+1, z=0)\}$,

(12) $h g\left(x \times r_{i}\right)=((x-t) /(s-t), i, 0)$ for $t \leqq x \leqq s$, $=((x-t) /(t-r), i, 0)$ for $r \leqq x \leqq t$,

(13) for any positive number $\varepsilon$ there is a number $\varepsilon^{\prime}$ such that $0<\varepsilon^{\prime}<\varepsilon$ and the pair of $\left.\operatorname{arcs}\left\{(x, y, z)|| x \mid=\varepsilon^{\prime}, 0 \leqq y \leqq k+1, z=0\right)\right\}$ lie in $h g(X)$.

Using the uniform continuity of $h^{-1}$, we find a positive number $\alpha$ small enough that $\alpha$-subsets of $E^{3}$ go to $\delta$-sets under $h^{-1}$.

If $t_{0}, t_{1}, t_{2}, \ldots, t_{k+1}$ is a sequence of numbers such that $1>t_{0}>\cdots>t_{k+1}=0$, then the following cells are associated with the $t_{i}$ 's: for $i=0, \ldots, k, D_{i}$ is the straight line segment from $\left(t_{i}, k+1-i, 0\right)$ to $\left(t_{i}, k+1,0\right) ; F_{i}$ is the line segment from $\left(t_{i}, k+1-i, 0\right)$ to $\left(t_{i+1}, k-i, 0\right) ; P_{i}$ is the 2-cell in the $x y$-plane bounded by the 
quadrilateral with vertices $\left(t_{i}, k+1,0\right),\left(t_{i}, k+1-i, 0\right),\left(t_{i+1}, k-i, 0\right)$ and $\left(t_{i+1}\right.$, $k+1,0) ; R_{i}$ is the 2-cell in the $x y$-plane bounded by the quadrilateral with vertices $(1, k+1-i, 0),(1, k-i, 0),\left(t_{i}, k+1-i, 0\right)$ and $\left(t_{i+1}, k-i, 0\right) ; G_{i}$ is the 2-cell in the $x y$-plane bounded by the quadrilateral with vertices $\left( \pm t_{i}, k+1-i, 0\right)$ and $\left( \pm t_{i+1}, k-i, 0\right) ; D_{i}^{\prime}, F_{i}^{\prime}, P_{i}^{\prime}$, and $R_{i}^{\prime}$ are the mirror images of the cells $D_{i}, F_{i}, P_{i}$, and $R_{i}$, respectively, on the other side of the plane $x=0 ; E_{i}=P_{i} \cup R_{i} ; E_{i}^{\prime}=P_{i}^{\prime} \cup R_{i}^{\prime}$; $N_{i}=\left\{(x, y, z) \mid t_{i+1}^{2}-x^{2} \leqq(y-k-1)^{2} \leqq t_{i}^{2}-x^{2}, y \geqq k+1, z=0\right\} ; T_{i}$ and $H_{i}$ are the solids of revolution obtained by revolving $N_{i} \cup P_{i} \cup P_{i}^{\prime}$ and $G_{i}$, respectively, about the $y$-axis; and $M=\bigcup T_{i}$.

Since the components of $([r, s] \times I)-X$ form a null sequence, it follows from (8), (9), and (13) that there is a sequence of numbers $t_{0}, t_{1}, \ldots, t_{k+1}$ such that

(14) $\alpha / 2>t_{0}>t_{1}>\cdots>t_{k+1}=0$,

(15) the arcs $D_{i}$ and $D_{i}^{\prime}$ lie in $h g(X)$,

(16) if $K$ is a component of $([r, s] \times I)-X$ such that $h g(\operatorname{Bd} K) \subset E_{i} \cup E_{i}^{\prime}$, then $h g(K) \cap\left(\bigcup\left\{T_{j}|| j-i \mid>1\right\}\right)=\varnothing$,

(17) $M \subset h(U)$.

There is a map $\mu$ of $E^{3}$ onto $E^{3}$ such that

(18) $\mu \mid E^{3}-M=$ identity,

(19) $\mu\left(T_{0}\right)=\mathrm{Cl}\left(M-\bigcup_{i=1}^{k} H_{i}\right)$,

(20) $\mu\left(T_{i}\right)=H_{i}$ for $i=1, \ldots, k$,

(21) $\mu h g(t \times I)=(0,0,0)$,

(22) $\mu \mid E^{3}-h g(t \times I)$ is a homeomorphism onto $E^{3}-(0,0,0)$.

The action of the map $\mu$ in the $x y$-plane is illustrated in Figure 1.

The required map $\beta=h^{-1} \mu h$. The numbers $u_{i}$ and $v_{i}$ are given by $\left(u_{i}, 0\right)=$ $g^{-1} h^{-1}\left(-t_{k+1-i}, 0,0\right)$ and $\left(v_{i}, 0\right)=g^{-1} h^{-1}\left(t_{k+1-i}, 0,0\right)$, and $\lambda$ is a homeomorphism of $[r, s] \times I$ onto $[r, s] \times I$ such that

$$
\begin{aligned}
\lambda \mid\left(\bigcup_{i}\left([r, s] \times r_{i}\right)\right) \cup(t \times I) & =\text { identity, } \\
\lambda\left(u_{i} \times\left[r_{i}, 1\right]\right) & =g^{-1} h^{-1}\left(D_{k+1-i}^{\prime}\right), \\
\lambda\left(v_{i} \times\left[r_{i}, 1\right]\right) & =g^{-1} h^{-1}\left(D_{k+1-i}\right) .
\end{aligned}
$$

It is straightforward to check that conditions (1) through (7) are satisfied.

Lemma 2. Suppose $E$ is a disk topologically embedded in $E^{3}, g$ is a map of $I^{2}$ $(=I \times I)$ onto $E$ such that $g(0 \times I)$ and $g(1 \times I)$ are distinct points in $\mathrm{Bd} E, g \mid(0,1) \times I$ is a homeomorphism onto $E-g(\{0,1\} \times I), U$ is an open set in $E^{3}$ containing $E-g(\{0,1\} \times I)$, and $\varepsilon>0$. Then there exist a partition $\left\{t_{i}\right\}$ of 1 , an $\varepsilon$-homeomorphism $\alpha$ of $I^{2}$ onto itself, and a map $\beta$ of $E^{3}$ onto $E^{3}$ such that

(1) $0=t_{0}<t_{1}<\cdots<t_{n}=1$ and $t_{i}-t_{i-1}<\varepsilon$,

(2) $\alpha \mid\{0,1\} \times I=$ identity,

(3) $\beta \mid E^{3}-U=$ identity,

(4) $\beta g \alpha\left(t_{i} \times I\right)$ is a point in $U$ for $i=0,1, \ldots, n$,

(5) $\beta g \alpha\left(t_{i} \times I\right) \neq \beta g \alpha\left(t_{j} \times I\right)$ if $i \neq j$, 


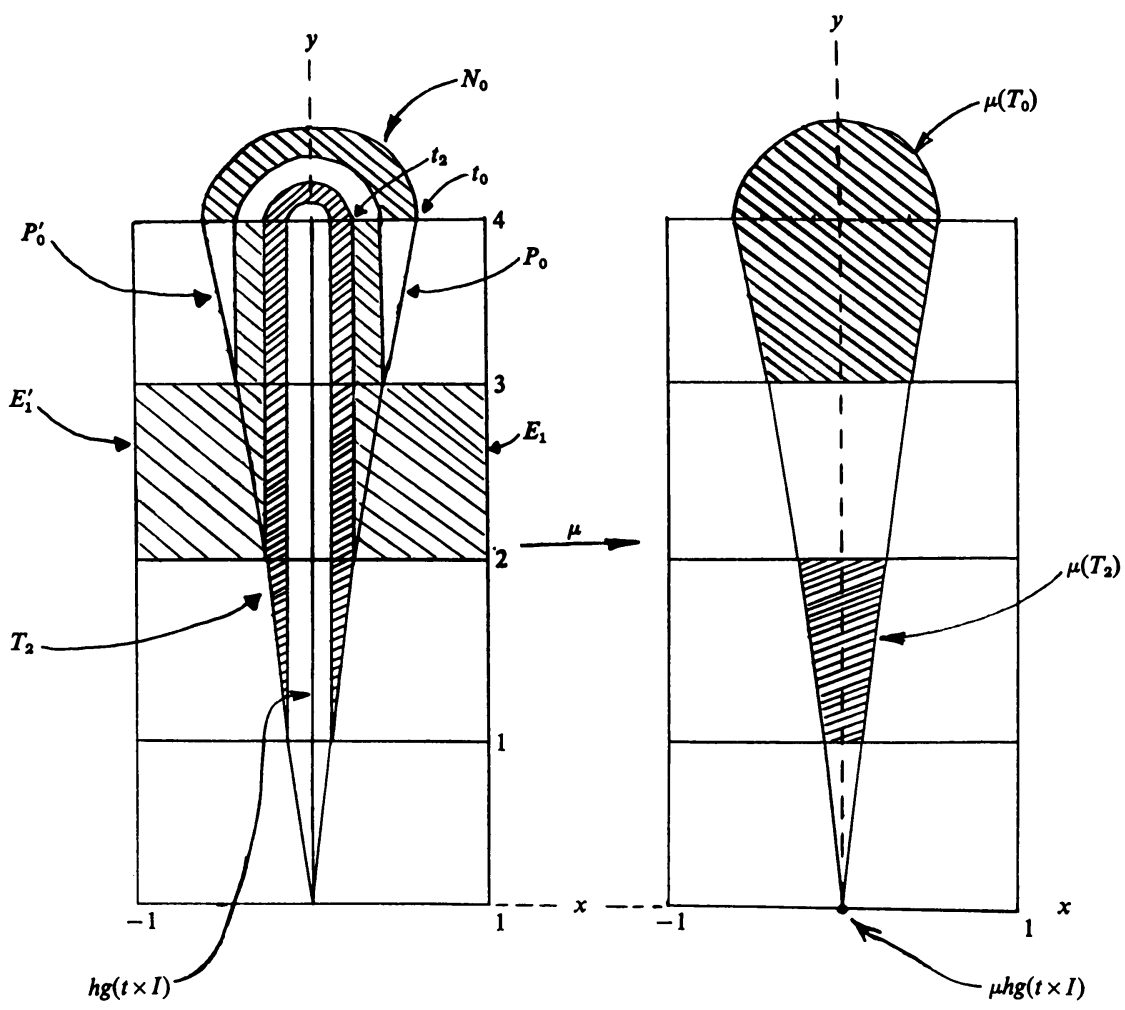

FIGURE 1

(6) $\beta \mid E^{3}-\bigcup_{i=1}^{n-1} g \alpha\left(t_{i} \times I\right)$ is a homeomorphism onto $E^{3}-\bigcup_{i=1}^{n-1} \beta g \alpha\left(t_{i} \times I\right)$,

(7) $\operatorname{Diam} \beta g \alpha\left(\left[t_{i-1}, t_{i}\right] \times I\right)<\varepsilon$ for $i=1, \ldots, n$.

Proof. The argument is given in two steps. In Step 1 the disk $g\left(I^{2}\right)=E$ is sliced into thin "vertical" strips by arcs, and these arcs are squeezed to points using Lemma 1. In Step 2 we find other arcs slicing the images of these strips into small disks. Enough care must be exercised in Step 1 to insure that the arcs of Step 2 can be realized as images of approximately vertical segments in $I^{2}$.

Step 1. Using Lemma 0 it is straightforward to show that there exist an $\varepsilon / 3-$ homeomorphism $\alpha_{1}$ of $I^{2}$ onto $I^{2}$, and an integer $k>3 / \varepsilon$ such that

(8) $\alpha_{1} \mid\{0,1\} \times I=$ identity,

(9) $g \alpha_{1}((i / 2 k) \times I)$ is tame for $i=1, \ldots,(2 k-1)$,

(10) $g \alpha_{1}([1 / 2 k,(2 k-1) / 2 k] \times i / 2 k)$ is tame for $i=1, \ldots,(2 k-1)$,

(11) Diam $g \alpha_{1}([0,1 / k] \times I)<\varepsilon$ and Diam $g \alpha_{1}([(k-1) / k, 1] \times I)<\varepsilon$,

(12) Diam $g \alpha_{1}([i / 2 k,(i+1) / 2 k] \times[j / 2 k,(j+1) / 2 k])<\varepsilon / 10$ for $i, j=1, \ldots,(2 k-2)$.

Let $B_{i}=(i / k) \times I$ and let $U_{1}, U_{2}, \ldots, U_{k-1}$ be disjoint open sets in $E^{3}$ such that

$$
g \alpha_{1}\left(B_{i}\right) \subset U_{i} \subset U \text { and } g \alpha_{1}(([0,(2 i-1) / 2 k] \cup[(2 i+1) / 2 k, 1]) \times I) \cap U_{i}=\varnothing .
$$

Define a homeomorphism $\mu$ of $I^{2}$ onto $I^{2}$ by $\mu((x, y))=(x, 1-y)$. In the statement 
of Lemma 1 take $U=U_{i}, t=i / k, r_{j}=j / 2 k,(j=1, \ldots, 2 k-1)$, and then apply Lemma 1 to the homeomorphism $g \alpha_{1} \mid[(2 i-1) / 2 k,(2 i+1) / 2 k] \times I$ if $i$ is an odd integer and to the homeomorphism $g \alpha_{1} \mu \mid[2 i-1 / 2 k, 2 i+1 / 2 k] \times I$ if $i$ is an even integer $(i=1,2, \ldots, k-1)$, thus obtaining maps $\lambda_{1}, \ldots, \lambda_{k-1}, \beta_{1}, \ldots, \beta_{k-1}$ satisfying the conclusions of Lemma 1.

The homeomorphisms $\lambda_{1}, \mu \lambda_{2} \mu^{-1}, \lambda_{3}, \mu \lambda_{4} \mu^{-1}, \lambda_{5}, \ldots$ are pieced together to obtain a homeomorphism $\alpha_{2}$ of $I \times I$ onto itself. Also, the maps $\beta_{1}, \ldots, \beta_{k-1}$ are pieced together to form a map $\beta^{\prime}$ of $E^{3}$ onto $E^{3}$. Note that

(13) $\alpha_{2} \mid \cup B_{i}=$ identity,

(14) $d\left(x, \alpha_{2}(x)\right)<\varepsilon / 3$,

(15) $\beta^{\prime} g \alpha_{1}\left(B_{i}\right)$ is a point in $U_{i}$,

(16) $\beta^{\prime} \mid E^{3}-\bigcup U_{i}=$ identity,

(17) $\beta^{\prime} \mid E^{3}-g \alpha_{1}\left(\bigcup B_{i}\right)$ is a homeomorphism onto $E^{3}-\beta^{\prime} g \alpha_{1}\left(\bigcup B_{i}\right)$.

Step 2. It follows that there are numbers $u(i, 1), \ldots, u(i, 2 k-1)$ and $v(i, 1), \ldots$, $v(i, 2 k-1)$ satisfying

$i / k<u(i, 1)<\cdots<u(i, 2 k-1)<(2 i+1) / 2 k<v(i, 1)<\cdots<v(i, 2 k-1)<(i+1) / k$

such that

(18) $g \alpha_{1} \alpha_{2}\left(A_{i j}\right)$ is tame, and

(19) Diam $\beta^{\prime} g \alpha_{1} \alpha_{2}(C)<\varepsilon$ for each component $C$ of

$$
I^{2}-\left(\bigcup_{i, j}\left(A_{i j} \cup B_{i}\right)\right),
$$

where the $A_{i j}$ 's are arcs defined by

$$
\begin{array}{r}
A_{i j}=\left(u(i, j) \times\left[r_{j}, 1\right]\right) \cup\left([u(i, j), v(i, j)] \times r_{j}\right) \cup\left(v(i, j) \times\left[0, r_{j}\right]\right) \\
\text { if } i \text { is an odd integer } \\
=\left(u(i, j) \times\left[0,1-r_{j}\right]\right) \cup\left([u(i, j), v(i, j)] \times\left(1-r_{j}\right)\right) \cup\left(v(i, j) \times\left[1-r_{j}, 1\right]\right) \\
\text { if } i \text { is an even integer. }
\end{array}
$$

There is a homeomorphism $\alpha_{3}$ of $I^{2}$ onto $I^{2}$ such that

(20) $d\left(x, \alpha_{3}(x)\right)<\varepsilon / 3$,

(21) $\alpha_{3} \mid B_{i}=$ identity,

(22) $\alpha_{3} \mid([0,1 / k] \cup[(k-1) / k, 1]) \times I=$ identity,

(23) $\alpha_{3}\left(t_{i j} \times I\right)=A_{i j}$ where $i / k<t_{i 1}<\cdots<t_{i, k-1}<i+1 / k$.

The required homeomorphism $\alpha=\alpha_{1} \alpha_{2} \alpha_{3}$; the map $\beta$ is $\beta^{\prime}$ followed by a map of $E^{3}$ onto $E^{3}$ that is the identity outside a small neighborhood of $\bigcup_{i, j} g \alpha\left(t_{i j} \times I\right)$ and takes the tame arcs $g \alpha\left(t_{i j} \times I\right)$ to distinct points. The map $\beta^{\prime}$ is illustrated in Figure 2.

The following is a stronger version of Theorem 2 .

THEOREM 2'. Suppose that $F$ is a 2-cell in the interior of a 3-manifold $M, g_{0}$ is a homeomorphism of $\Delta_{2}$ onto $F, U$ is an open subset of $M$ containing $F-g_{0}\left(\mathrm{Bd} \Delta_{1}\right)$, and 


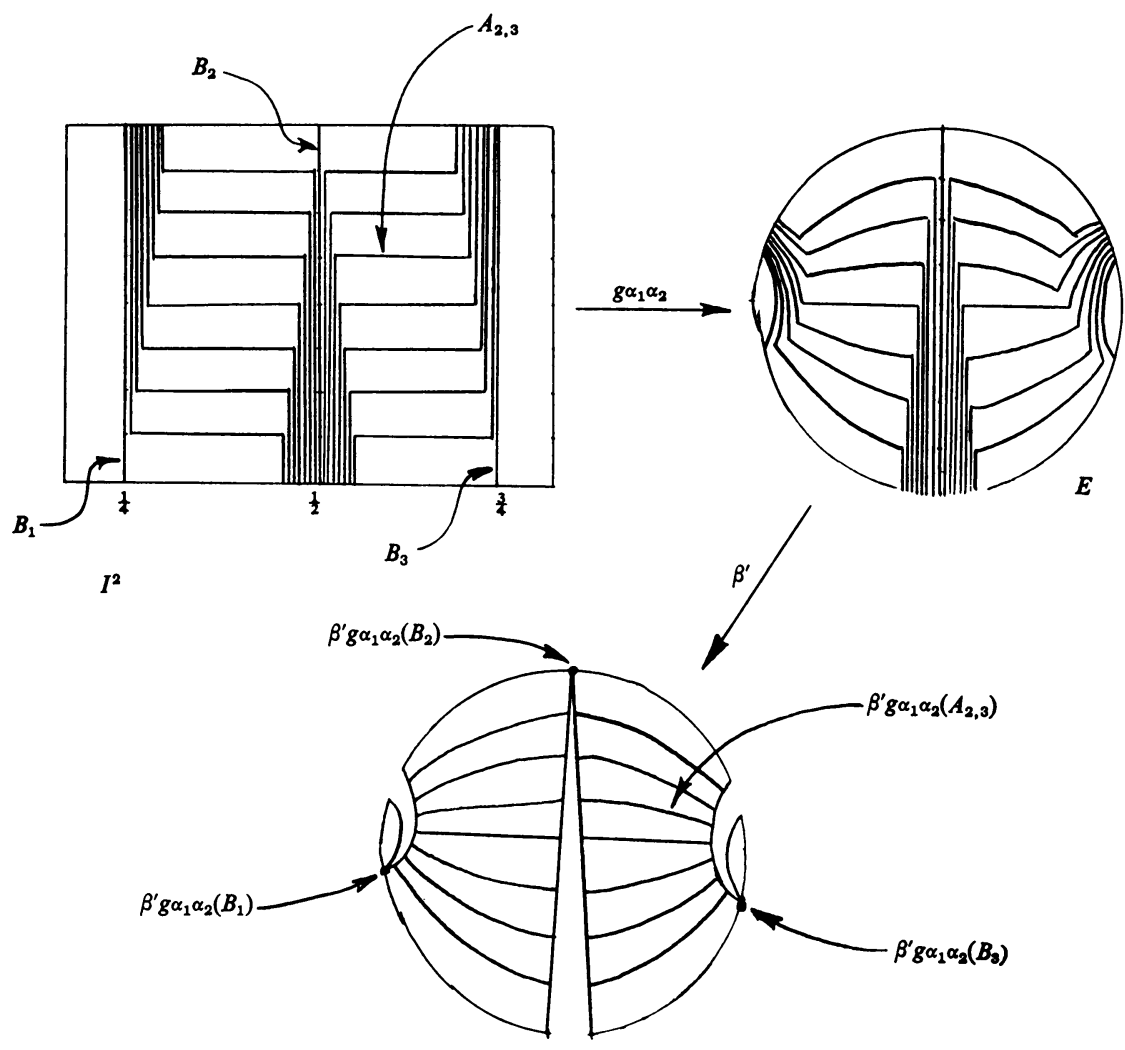

FIGURE 2

$\varepsilon>0$. Then there exist a map fof $M$ onto $M$, a homeomorphism $g$ of $\Delta_{2}$ onto $F$, and a homeomorphism $h$ of $\Delta_{1}$ onto $f(F)$ such that

(1) $f$ is a homeomorphism of $M-F$ onto $M-f(F)$,

(2) $f \mid M-U=$ identity,

(3) $f g=h \pi$,

(4) $d\left(g_{0}, g\right)<\varepsilon$,

(5) $g\left|\operatorname{Bd} \Delta_{1}=g_{0}\right| \operatorname{Bd} \Delta_{1}$.

Proof. Let $P$ be a map of $I^{2}$ onto $\Delta_{2}$ so that $P(0 \times I)=(-1,0,0), P=(1 \times I)$ $=(1,0,0), P \mid(0,1) \times I$ is a homeomorphism onto $\Delta_{2}-\{(-1,0,0),(1,0,0)\}$ and $P(t \times I)=\pi^{-1}(r)$ for some $r \in \Delta_{1}$. The maps $g$ and $f$ are obtained as limits of sequences of homeomorphisms $\left\{g_{n}\right\}$ and maps $\left\{f_{n}\right\}$ respectively.

Let $U_{0}, U_{1}, \ldots$ be a sequence of bounded open sets in $E^{3}$ such that $U \supset U_{0} \supset U_{1} \ldots$ and $\cap U_{i}=F$, and let $\varepsilon_{0}, \varepsilon_{1}, \ldots$ be a sequence of positive numbers such that

(6) $\sum_{0}^{\infty} \varepsilon_{i}<\varepsilon$.

Let $f_{0}$ be the identity map of $E^{3}$ onto $E^{3}$, and let $V_{0}$ be a connected open set so that $F \subset V_{0} \subset U_{0}$. Apply Lemma 2 to the 2-cell $F$, open set $V_{0}$, and map $g_{0} P$ to obtain maps $\alpha_{0}$ and $\beta_{0}$ satisfying the conclusions of Lemma 2. Let $g_{1}=g_{0} P \alpha_{0} P^{-1}$ 
and $f_{1}=\beta_{0} f_{0}$. Note that conditions (4) and (6) of Lemma 2 imply that there is a set $N_{1}=\{t(1, i)\}$ of $k_{1}+1$ points in $I$ such that $0=t(1,0)<t(1,1)<\cdots<t\left(1, k_{1}\right)=1$ and $f_{1} g_{1} P(t \times I)$ is a point if and only if $t \in N_{1}$. Define

$$
F(1, i)=f_{1} g_{1} P([t(1, i-1), t(1, i)] \times I) .
$$

By applying Lemma 2 with a small enough epsilon we insure that Diam $F(1, i)<\varepsilon_{0} / 2$ and $d\left(g_{0}, g_{1}\right)<\varepsilon_{0}$. There is an open set $V_{1}$ contained in $f_{1}\left(U_{1}\right)$ with exactly $k_{1}$ components $V(1,1), \ldots, V\left(1, k_{1}\right)$ such that

$$
F(1, i)-f_{1} g_{1} P(\{t(1, i-1), t(1, i)\} \times I) \subset V(1, i)
$$

and Diam $V(1, i)<\varepsilon_{0}$.

We proceed inductively. Assume that a set $N_{n-1}=\{t(n-1, i) \mid 0=t(n-1,0)<\cdots$ $\left.<t\left(n-1, k_{n-1}\right)=1\right\}$ of $k_{n-1}+1$ points in $I$, an open set $V_{n-1}$ with components $V(n-1,1), \ldots, V\left(n-1, k_{n-1}\right), 2$-cells

$$
F(n-1, i)=f_{n-1} g_{n-1} P([t(n-1, i-1), t(n-1, i)] \times I),
$$

and maps $g_{n-1}$ and $f_{n-1}$ have been defined. For $i=1, \ldots, k_{n-1}$ apply Lemma 2 to the 2-cell $F(n-1, i)$, open set $V(n-1, i)$ and map

$$
f_{n-1} g_{n-1} P \mid[t(n-1, i-1), t(n-1, i)] \times I
$$

and obtain maps $\alpha_{n-1}^{i}$ and $\beta_{n-1}^{i}$. The maps $\alpha_{n-1}^{i}\left(i=1, \ldots, k_{n-1}\right)$ are pieced together to obtain a homeomorphism $\alpha_{n-1}$ from $I^{2}$ onto $I^{2}$ such that

$$
\alpha_{n-1} \mid[t(n-1, i-1), t(n-1, i)] \times I=\alpha_{n-1}^{i} .
$$

Let $g_{n}=g_{n-1} P \alpha_{n-1} P^{-1}$, and define the map $f_{n}$ so that

$$
\begin{aligned}
& f_{n}\left|E^{3}-f_{n-1}^{-1}\left(V_{n-1}\right)=f_{n-1}\right| E^{3}-f_{n-1}^{-1}\left(V_{n-1}\right) \\
& f_{n}\left|f_{n-1}^{-1}(V(n-1, i))=\beta_{n-1}^{i} f_{n-1}\right| f_{n-1}^{-1}(V(n-1, i)) .
\end{aligned}
$$

Conditions (4) and (6) of Lemma 2 imply that there is a set $N_{n}=\{t(n, i)\}$ of $k_{n}+1$ points in $I$ such that $0=t(n, 0)<\cdots<t\left(n, k_{n}\right)=1$, and $f_{n} g_{n} P(t \times I)$ is a point if and only if $t \in N_{n}$. Define

$$
F(n, i)=f_{n} g_{n} P([t(n, i-1), t(n, i)] \times I) .
$$

By applying Lemma 2 with a small enough epsilon we insure that $\operatorname{Diam} F(n, i)$ $<\varepsilon_{n-1} / 2$ and

(7) $d\left(g_{n-1}, g_{n}\right)<\varepsilon_{n-1}$.

There is an open set $V_{n}$ contained in $f_{n}\left(U_{n}\right)$ with exactly $k_{n}$ components $V(n, 1), \ldots$, $V\left(n, k_{n}\right)$ such that $\operatorname{Diam} V(n, i)<\varepsilon_{n-1}, F(n, i) \subset V(n, i) \cup f_{n} g_{n} P(\{t(n, i-1), t(n, i)\} \times I)$, and

(8) $V(n, i) \subset V(n-1, j)$ for some $j$.

We further suppose that

(9) $\sum_{n}^{\infty} \varepsilon_{i}$ is so small that if $d\left(x_{1}, x_{2}\right)>1 / n$ for $x_{1}, x_{2} \in \Delta_{2}$ then

$$
d\left(g_{n}\left(x_{1}\right), g_{n}\left(x_{2}\right)\right)>2 \sum_{n}^{\infty} \varepsilon_{i} \text {. }
$$


We also have that

(10) $d\left(f_{n}, f_{n+1}\right)<\varepsilon_{n-1}$ if $n \neq 0$,

(11) $f_{n}\left|E^{3}-U_{n}=f_{n+1}\right| E^{3}-U_{n}$,

(12) $f_{n} \mid E^{3}-g_{n} P\left(N_{n} \times I\right)$ is a homeomorphism onto $E^{3}-f_{n} g_{n} P\left(N_{n} \times I\right)$,

(13) $N_{1} \subset N_{2} \subset \cdots$ and $\bigcup N_{i}$ is dense in $I$,

(14) $f_{n} g_{n} P\left|N_{n} \times I=f_{n+1} g_{n+1} P\right| N_{n} \times I$,

(15) $f_{n} g_{n} P(t \times I)$ is a point if $t \in N_{n}$,

(16) $f_{n} g_{n} P(t \times I) \cap f_{n} g_{n} P(r \times I)=\varnothing$ if $t, r \in I$ and $t \neq r$,

(17) Diam $f_{n} g_{n} P(t \times I)<\varepsilon_{n-1}$ if $t \in I$,

(18) if $t, r \in I-\bigcup N_{i}$ and $t \neq r$, then there are numbers $n, i$ and $j$ with $j \neq i$ such that $f_{n} g_{n} P(t \times I) \subset V(n, i), f_{n} g_{n} P(r \times I) \subset V(n, j)$,

(19) $f_{n+1} f_{n}^{-1}(V(n, i)) \subset V(n, i)$.

We let $g=\operatorname{limit} g_{n}$ and $f=\operatorname{limit} f_{n}$. That $g$ and $f$ are maps follows from (6), (7), and (10). It follows from (6), (7), (9) and [7, Theorem 7] that $g$ is a homeomorphism of $\Delta_{2}$ onto $F$ such that $d\left(g_{0}, g\right)<\varepsilon$. Conditions (11) and (12) imply that $f \mid E^{3}-F$ is a homeomorphism onto $E^{3}-f(F)$ and conditions (8) and (13)-(19) imply $f g \pi^{-1}=h$ is a homeomorphism of $\Delta_{1}$ onto $f(F)$.

5. Applications to cubes with handles. In this section we extend the results of $\S 3$ to embeddings of cubes with handles.

THEOREM 10. Suppose that $H_{n}$ is a cube with $n$ handles in the interior of a 3manifold $M$, and $U$ is an open subset of $M$ containing $H_{n}$. Let $D_{n}$ be a disk with $n$ holes, and let $\pi_{1}$ be the projection of $D_{n} \times I$ onto $D_{n}$. Then there exist a map $f$ of $M$ onto itself, a homeomorphism $h$ of $D_{n}$ onto $f\left(H_{n}\right)$, and a homeomorphism $g$ of $D_{n} \times I$ onto $H_{n}$ such that

(1) $f$ is a homeomorphism of $M-H_{n}$ onto $M-f\left(H_{n}\right)$,

(2) $f \mid M-U=$ identity,

(3) $f g=h \pi_{1}$.

The same techniques used to prove Theorem 1 may be reapplied to prove Theorem 10.

THEOREM 11. Suppose $D_{n}$ is a disk with $n$ holes in the interior of a 3-manifold $M$, and $U$ is an open subset of $M$ containing $D_{n}$. Then there exists a map $f$ of $M$ onto itself such that

(1) $f$ is a homeomorphism of $M-D_{n}$ onto $M-f\left(D_{n}\right)$,

(2) $f \mid M-U=$ identity,

(3) $f\left(D_{n}\right)$ is a wedge of $n$ simple closed curves.

Furthermore, $f$ may be obtained so that the preimage of a point of $f\left(D_{n}\right)$ is either an arc or a 2 -frame.

Proof. Let $J_{0}, J_{1}, \ldots, J_{n}$ be the boundary components of $D_{n}$. Using Lemma 0 we find a tame $n$-frame $G^{\prime}$ in $D_{n}$ such that $G^{\prime} \cap \operatorname{Bd} D_{n}=\operatorname{Bd} G^{\prime}, \operatorname{Bd} G^{\prime} \subset J_{0}$, and each 
component of $D_{n}-G^{\prime}$ contains (exactly) one of the curves $J_{1}, \ldots, J_{n}$. Then $G^{\prime}$ is contained in a tame $2 n$-frame $G$ in $D_{n}$ such that $G \cap \mathrm{Bd} D_{n}=\mathrm{Bd} G$ and each of the boundary curves $J_{1}, \ldots, J_{n}$ meets one of the arcs of $G-G^{\prime}$.

Since $G$ is tame, there exists a map $f_{1}$ of $M$ onto itself such that $f_{1} \mid M-U=$ identity, $f_{1}$ takes $G$ to a point, and $f_{1}$ is a homeomorphism of $M-G$ onto $M-f_{1}(G)$. Consequently, $f_{1}\left(D_{n}\right)$ is the union of pinched annuli $A_{1}, \ldots, A_{n}$ joined at a point $p$.

We select a tame $\operatorname{arc} \alpha_{i}$ spanning $A_{i}$ whose endpoints lie in distinct components of Bd $A_{i}-p(i=1, \ldots, n)$. There is a map $f_{2}$ of $M$ onto itself such that $f_{2} \mid M-U$ $=$ identity, $f_{2}$ takes each arc $\alpha_{i}$ to a point of $M$, and $f_{2}$ is a homeomorphism of $M-\bigcup \alpha_{i}$ onto $M-f_{2}\left(\cup \alpha_{i}\right)$. Then $f_{2} f_{1}\left(D_{n}\right)$ is the union of disks $E_{1}, \ldots, E_{2 n}$, where the intersection of any pair of the $E$ 's is either one point or two points in the boundary of each.

Applying Theorem $2^{\prime}$ to each of the $E$ 's, we find that there exist a map $f_{3}$ of $M$ onto itself, homeomorphisms $g_{i}$ of $\Delta_{2}$ onto $E_{i}$, and homeomorphisms $h_{i}$ of $\Delta_{1}$ onto $f_{3}\left(E_{i}\right)$ such that

(1) $f_{3}$ is a homeomorphism of $M-f_{2} f_{1}\left(D_{n}\right)$ onto $M-f_{3} f_{2} f_{1}\left(D_{n}\right)$,

(2) $f_{3} \mid M-U=$ identity,

(3) $f_{3}\left(E_{j}\right) \cap f_{3}\left(E_{k}\right)=E_{j} \cap E_{k}$, whenever $j \neq k$,

(4) $f_{3} g_{i}=h_{i} \pi(i=1, \ldots, 2 n)$.

The composition $f_{3} f_{2} f_{1}$ produces the required map $f$.

COROllaRY 12. Suppose $H_{n}$ is a cube with $n$ handles in the interior of a 3-manifold $M$, and $U$ is an open subset of $M$ containing $H_{n}$. Then there exists a map fof $M$ onto itself such that

(1) $f$ is a homeomorphism of $M-H_{n}$ onto $M-f\left(H_{n}\right)$,

(2) $f \mid M-U=$ identity,

(3) $f\left(H_{n}\right)$ is a wedge of $n$ simple closed curves.

We conclude this section by showing why an arbitrary simple closed curve cannot be enlarged to a solid torus that projects onto the curve. Let $J$ denote the simple closed curve that pierces no disk of [5].

THEOREM 13. There is no solid torus $T$ in $E^{3}$ such that $E^{3}-T$ and $E^{3}-J$ are homeomorphic.

We require the following definitions: if $T$ is a solid torus and $J$ is a tame simple closed curve in Int $T$, the order of $T$ with respect to $J$ is the minimal number of points of intersection of a meridianal disk of $T$ with the curve $J$ (for all meridianal disks of $T$ ), and is denoted $O(T, J)$; if $T$ and $T^{\prime}$ are tame solid tori with $T^{\prime} \subset \operatorname{Int} T$, then the order of $T$ with respect to $T^{\prime}$, denoted $O\left(T, T^{\prime}\right)$, is defined to be the order of $T$ with respect to a center line of $T^{\prime}$. This definition of $O\left(T, T^{\prime}\right)$ is independent of the choice of center line of $T^{\prime}[18$, p. 172].

Proof of Theorem 13. Suppose $T$ is a solid torus in $E^{3}$ such that there exists a homeomorphism $h$ of $E^{3}-J$ onto $E^{3}-T$. Consider the sequence of polyhedral 
solid tori $T_{1}, T_{2}, \ldots$ used by Bing in the definition of $J$. Since the fundamental group of $h\left(E^{3}-T_{i}\right)$ is not infinite cyclic, the closure $C_{i}$ of the component of $E^{3}-h\left(\operatorname{Bd} T_{i}\right)$ containing $T$ is a tame solid torus $(i \geqq 2)$. Let $H$ be a tame solid torus in Int $T$ concentric with $T$, and let $k$ be a positive integer. It is easy to show that $O\left(T_{n}, T_{n+k}\right)$ $=O\left(C_{n}, C_{n+k}\right)$ and that $O\left(C_{n}, H\right)>0$ for sufficiently large integers $n$. Furthermore, Step 3 of [5] indicates that $O\left(T_{n}, T_{n+k}\right) \geqq 2^{k}$. It follows from the product formula of Schubert $[18$, p. 175$]$ that

$$
O\left(C_{n}, H\right)=O\left(C_{n}, C_{n+k}\right) \cdot O\left(C_{n+k}, H\right) \geqq 2^{k} \cdot O\left(C_{n+k}, H\right) .
$$

Consequently, if $n$ is sufficiently large, $O\left(C_{n}, H\right)$ must be infinite. This is impossible.

Question. Does there exist an arc $A$ in $E^{3}$ such that for each 3-cell $K$ in $E^{3}$, the complement of $K$ is not homeomorphic to the complement of $A$ ?

\section{REFERENCES}

1. W. R. Alford, "Some 'nice' wild 2-spheres in $E^{3}$ " in Topology of 3-manifolds and related topics, Prentice-Hall, New York, 1962, pp. 29-33.

2. J. J. Andrews and M. L. Curtis, $n$-space modulo an arc, Ann. of Math. (2) 75 (1962), 1-7.

3. S. Armentrout, Concerning a wild cell of Bing, Duke Math. J. 33 (1966), 689-704.

4. S. Armentrout, L. L. Lininger and D. V. Meyer, Equivalent decompositions of $E^{3}$, Pacific J. Math. 24 (1968), 205-227.

5. R. H. Bing, A simple closed curve that pierces no disk, J. Math. Pures Appl. (9) 35 (1956), 337-343.

6. - A surface is tame if its complement is 1-ULC, Trans. Amer. Math. Soc. 101 (1961), 294-305.

7. - Each disk in $E^{3}$ contains a tame arc, Amer. J. Math. 84 (1962), 583-590.

8. R. H. Bing and A. Kirkor, An arc is tame in 3-space if and only if it is strongly cellular, Fund. Math. 55 (1964), 175-180.

9. J. L. Bryant, Euclidean space modulo a cell, Notices Amer. Math. Soc. 14 (1967), 916.

10. R. F. Craggs, Improving the intersection of polyhedra in 3-manifolds, Illinois J. Math. 12 (1968), 567-586.

11. R. J. Daverman and W. T. Eaton, $A$ dense set of sewings of two crumpled cubes yields $S^{3}$, Fund. Math. 65 (1969), 51-60.

12. R. H. Fox and E. Artin, Some wild cells and spheres in three-dimensional space, Ann. of Math. (2) 49 (1948), 979-990.

13. N. Hosay, The sum of a real cube and a crumpled cube is $S^{3}$, Notices Amer. Math. Soc. 10 (1963), 666; see also Errata 11 (1964), 152.

14. K. W. Kwun, Product of Euclidean spaces modulo an arc, Ann. of Math. (2) 79 (1964), 104-107.

15. L. L. Lininger, Some results on crumpled cubes, Trans. Amer. Math. Soc. 118 (1965), 534-549.

16. D. R. McMillan, Jr., A criterion for cellularity in a 3-manifold. II, Trans. Amer. Math. Soc. 126 (1967), 217-224.

17. D. V. Meyer, $E^{3}$ modulo a 3-cell, Pacific J. Math. 13 (1963), 193-196.

18. H. Schubert, Knoten und Vollringe, Acta. Math. 90 (1953), 131-286.

UNIVERSITY OF TENNESSEE,

KNOXVIlle, TenNessee 